\title{
QUARK MASS EFFECTS AND ANOMALIES
}

\author{
R. AKHOURY \\ Randall Laboratory of Physics, University of Michigan, Ann Arbor, MI 48109, USA
}

and

F.J. YNDURÁIN

Departamento de Fisica Teorica, Universidad Autonoma de Madrid, E-28049 Madrid, Spain

Received 5 February 1988

\begin{abstract}
When one takes into account that different quarks have unequal masses, five-point functions associated with pentagon graphs with two external gluons $(2 \mathrm{~g})$ are modified by terms involving quark mass ratios. In this context we prove an exact low energy theorem for the amplitudes $(2 \mathrm{~g}) \rightarrow \mathrm{rj} \ell$ with $\mathrm{r}, \mathrm{j}, \ell$ in the octet of pseudoscalar Goldstone bosons. A possible manifestation of these effects could be an enhancement of processes $(2 g) \rightarrow K \bar{K} \pi$ over $(2 g) \rightarrow \eta \pi \pi$ ones as indeed are seen experimentally in $ı$ decays. A contribution to $\eta^{\prime} \rightarrow \eta \pi \pi$ is also found.
\end{abstract}

\section{Introduction}

It has been known for a long time [1] that axial currents have triangle, square and pentagon anomalies [1,2], which can be traced to the divergences of the triangle graph. For example, and concentrating on gluon anomalies [3] ${ }^{\# 1}$, the current $A_{\mathrm{qq}}^{\mu}=\overline{\mathrm{q}} \gamma^{\mu} \gamma_{5} \mathrm{q}$ has a triangle (gluon) anomaly, so that

$\partial_{\mu} A_{\mathrm{qq}}^{\mu}=\left(T_{\mathrm{F}} \alpha_{\mathrm{s}} / 2 \pi\right) \tilde{G} G+2 \mathrm{i} m_{\mathrm{q}} \overline{\mathrm{q}} \gamma_{5} \mathrm{q}$,

where $T_{\mathrm{F}}=\frac{1}{2}$ is defined by $\operatorname{Tr} t^{a} t^{b}=T_{\mathrm{F}} \delta_{a b}, t^{a}$ the generators of colour SU( 3$)$ and

$G_{a}^{\mu \nu}=\partial^{\mu l} B_{a}^{\nu}-\partial^{\nu} B_{a}^{\mu}+g f_{a b c} B_{b}^{\mu} B_{c}^{\nu}, \quad \widetilde{G}_{a}^{\mu \nu}=\frac{1}{2} \epsilon^{\mu \nu \alpha \beta} G_{a \alpha \beta}$.

Moreover, the anomaly does not get renormalized [4] which leads to low energy theorems, the best known of which is that giving the $\pi^{0} \rightarrow 2 \gamma$ decay amplitude via the (photon) anomaly and PCAC.

The analysis of low energy theorems related to anomalies is usually carried neglecting the mass differences of the (light, $u, d, s$ ) quarks. While for triangle and square anomalies this is likely to be a valid procedure (to corrections $\mathrm{O}\left(\mathrm{m}_{\mathrm{q}} /\right.$ typical hadronic mass $)$ ) the situation is different for five-point functions. Loosely speaking, we have the following: let

$\left.F\left(\mathrm{~g}_{1}^{\alpha}\left(p_{1}\right) \mathrm{g}_{2}^{h}\left(p_{2}\right)\right) \rightarrow \mathrm{r}\left(k_{1}\right) \mathrm{j}\left(k_{2}\right) \ell\left(k_{3}\right)\right)$

be the amplitude for the gluons $\mathrm{g}_{1} \mathrm{~g}_{2}$ into the pseudoscalar mesons $\mathrm{r}, \mathrm{j}, \ell ; p, k$ are the momenta, $a, b$, the color indices of the gluons. For the pentagon case, we have sufficiently many momenta that we can take two $k$ 's to zero and still get a nonzero value for $F$. With standard current algebra techniques, each zero momentum is seen to give a commutator and we thus get double commutators which are nonzero for nonvanishing quark masses. The remainder is an anomalous triangle, which can be evaluated exactly in the limit of small momenta. The net

\#I The extension to photon anomalies, together with more details on the present work will be presented in ref. [3]. 
result is a low energy theorem for $F$ expressed in terms of the anomaly times coefficients depending on quark mass ratios, $m_{\mathrm{q}} / m_{\mathrm{q}}$, hence nonvanishing in the chiral limit. For example, if $\mathrm{r}=\mathrm{K}^{+}, \mathrm{j}=\overline{\mathrm{K}}^{0}, \ell=\pi^{-}$we obtain that the effective lagrangian

$\mathscr{L}_{\text {int }}^{\text {eff }}\left(\mathrm{K}^{+} \overline{\mathrm{K}}^{0} \pi^{-}\right)=\left(T_{\mathrm{F}} \alpha_{\mathrm{s}} / 8 \pi f_{\pi}^{3}\right) \lambda\left(\mathrm{K}^{+} \overline{\mathrm{K}}^{0} \pi^{-}\right) \phi_{\mathrm{K}^{+}} \phi_{\overline{\mathrm{K}}^{0}} \phi_{\pi^{-}} \widetilde{G} G$,

$\lambda\left(\mathrm{K}^{+} \overline{\mathrm{K}}^{0} \pi^{-}\right)=\frac{\left(m_{\mathrm{s}}+m_{\mathrm{d}}\right)\left(m_{\mathrm{s}}+m_{\mathrm{u}}\right)\left(m_{\mathrm{d}}+m_{\mathrm{u}}\right)}{2 \sqrt{2} m_{\mathrm{s}} m_{\mathrm{d}} m_{\mathrm{u}}} \frac{f_{\pi}^{2}}{f_{\mathrm{K}}^{2}}$

gives $F\left(g_{1}+\mathrm{g}_{2} \rightarrow \mathrm{K}^{+} \overline{\mathrm{K}}^{0} \pi^{-}\right)$correct to $\mathrm{O}\left((p, k)^{4}\right)$; and neglecting higher order flavor $\mathrm{SU}(3)$ corrections. (Eqs. (3) appear to be singular if one of the quark masses vanish. This is not so as will be discussed below.)

We have not been able to find an application of our results as clear and precise as the calculation of $\pi^{0} \rightarrow 2 \gamma$ using the standard anomaly; but a possible phenomenological implication would be for a rough calculation of the widths and partial rates of the $1(1440)$ glueball, and the width of the $\eta^{\prime}(960)$, in qualitative agreement with experiment. (Note that the two first are hitherto unexplained phenomena; for the first applying naive Zweig rule considerations [5] ${ }^{\# 2}$, we would expect a width $\Gamma_{i} \sim 0.3-1 \mathrm{MeV}$, two orders of magnitude smaller than the experimental figure).

\section{Low energy theorem}

Consider the axial currents $A_{\mathrm{r}}^{\mu}, A_{\mathrm{j}}^{\nu}, A_{\ell}^{\lambda}$ associated with particles of the octet of pseudoscalar Goldstone mesons; if, e.g., $\mathrm{r}=\mathrm{K}^{+}, \mathrm{j}=\overline{\mathrm{K}}^{0}, \ell=\pi^{-}$,

$A_{\mathrm{r}}^{\mu}=\overline{\mathrm{u}} \gamma^{\mu} \gamma_{5} \mathrm{~s}, \quad A_{\mathrm{j}}^{\nu}=-\overline{\mathrm{s}} \gamma^{\nu} \gamma_{5} \mathrm{~d}, \quad A_{\ell}^{\lambda}=\overline{\mathrm{d}} \gamma^{\lambda} \gamma_{5} \mathrm{u}$.

The field operators themselves will be denoted by $\phi_{\mathrm{r}}, \phi_{\mathrm{j}}, \phi_{\mathrm{Q}}$. We will use the PCAC definitions $\phi_{i}=$ $\left(1 / \sqrt{2} f_{i} M_{i}^{2}\right) \partial A_{i}$, with $f_{\pi} \simeq 92 \mathrm{MeV}$. Since none of the $A_{i}$ have anomalies we may also write, if $A_{i}^{\alpha}=\overline{\mathrm{q}}_{1} \gamma^{\alpha} \gamma_{5} \mathrm{q}_{2}$, $\partial A_{i}=\mathrm{i}\left(m_{1}+m_{2}\right) \overline{\mathrm{q}}_{1} \gamma_{5} \mathrm{q}_{2}$, with $m$ the quark masses. If $e_{i}\left(p_{i}\right)$ are the polarization vectors of the gluons, we can use gauge invariance to write the amplitude (2) as

$F=\left(F_{2}^{\alpha \beta}+F_{4}^{\alpha \beta}\right) e_{1 \alpha}\left(p_{1}\right) e_{2 \beta}\left(p_{2}\right) \delta_{a b}$,

$F_{2}^{\alpha \beta}=\Phi\left(p_{1}, \ldots, k_{3}\right) \epsilon^{\alpha \beta \mu \nu} p_{1 \mu} p_{2 \nu}, \quad F_{4}^{\alpha \beta}=\Psi\left(p_{1}, \ldots, k_{3}\right) \epsilon^{\alpha \lambda \mu \nu} p_{1 \lambda} k_{i \mu}\left(g_{\nu}^{\beta} p_{1} p_{2}-p_{1}^{\beta} p_{2 \nu}\right)+$ permutations.

Note that $F_{2}$ is $\mathrm{O}\left((p, k)^{2}\right)$, and $F_{4}$ is $\left(\mathrm{O}(p, k)^{4}\right)$. By using the reduction formulas, we may consider the $F$ to be defined for arbitrary values of the momenta. The existence of Adler's zeros [6] implies that $\Phi$ must vanish when one of the $k$ vanishes, and the others are on the mass shell, so if we write

$\Phi\left(p_{1}, \ldots, k_{3}\right)=\left(2-k_{\mathrm{r}}^{2} / M_{\mathrm{r}}^{2}-k_{\mathrm{j}}^{2} / M_{\mathrm{j}}^{2}-k_{\ell}^{2} / M_{\ell}^{2}\right) \Phi\left(p_{1}, \ldots, k_{3}\right)$,

then $\Phi$ is free from zeros. It is also free from poles to leading order in non-chiral SU( 3 ) breaking, so in this limit it will be a smooth function of the momenta. The physical value of $\Phi$ is for all particles on their mass shells, i.e., $\Phi_{\text {phys }}=-\hat{\Phi}$. Expanding in powers of the momenta, $\hat{\Phi}=\hat{\Phi}(0)+\mathrm{O}\left((p, k)^{2}\right) ; \Phi(0)=2 \hat{\Phi}(0)$. We thus find that, to corrections $\mathrm{O}\left((p, k)^{4}\right)$, the physical scattering amplitude is given by

$F_{\text {phys }} \simeq F_{2 \mathrm{phys}}^{\alpha \beta} e_{1 \alpha} e_{2 \beta} \delta_{a b}, \quad F_{2 \mathrm{phys}}^{\alpha \beta}=-\frac{1}{2} \Phi(0) \epsilon^{\alpha \beta \mu \nu} p_{1 \mu} p_{2 \nu}$.

Our task is to evaluate $\Phi(0)$. Using the PCAC definitions and reduction formulas, and neglecting terms $\mathrm{O}\left((p, k)^{4}\right)$,

\#2 The result is obtained by comparing with the decay of $\eta_{\mathrm{c}}, \Gamma\left(\eta_{\mathrm{c}}\right) \simeq 11 \mathrm{MeV}$. The only difference is the replacement of $C_{\mathrm{F}} \alpha_{\mathrm{s}}\left(M_{\mathrm{\eta}_{\mathrm{k}}}^{2}\right)$ by $T_{\mathrm{F}} \alpha_{\mathrm{s}}\left(M_{\mathrm{l}}^{2}\right)$, and phase space. The spread from 0.3 to $1 \mathrm{MeV}$ for $\Gamma(1)$ depends upon the relative weight of the various decay channels for t. 
$F=\frac{i^{3}}{2^{3 / 2} f_{\mathrm{r}} f_{\mathrm{j}} f_{\ell}} \frac{(2 \pi)^{4}}{(2 \pi)^{9 / 2}} \int \mathrm{d} x \mathrm{~d} y \exp \left[\mathrm{i}\left(k_{1} \cdot x+k_{2} \cdot y\right)\right]\left\langle 0\left|\mathrm{~T} \partial A_{\mathrm{r}}(x) \partial A_{\mathrm{j}}(y) \partial A_{\mathrm{l}}(0)\right| \mathrm{g}_{1} \mathrm{~g}_{2}\right\rangle$.

To calculate the RHS we consider

$R_{\mathrm{rj} \ell}^{\mu}=\int \mathrm{d} x \mathrm{~d} y \exp \left[\mathrm{i}\left(k_{1} \cdot x+k_{2} \cdot y\right)\right]\left\langle 0\left|\mathrm{~T} A_{\mathrm{r}}^{\mu}(x) \partial A_{\mathrm{j}}(y) \partial A_{\ell}(0)\right| \mathrm{g}_{1} \mathrm{~g}_{2}\right\rangle$,

contracting with $-\mathrm{i} k_{1 \mu}$ we find

$$
\begin{aligned}
& -\mathrm{i} k_{1 \mu} R_{\mathrm{rj} \ell}^{\mu}=\int \mathrm{d} x \mathrm{~d} y \exp \left[\mathrm{i}\left(k_{1} \cdot x+k_{2} \cdot y\right)\right]\left\langle 0\left|\mathrm{~T} \partial A_{\mathrm{r}}(x) \partial A_{\mathrm{j}}(y) \partial A_{\ell}(0)\right| \mathrm{g}_{1} \mathrm{~g}_{2}\right\rangle \\
& \quad+\int \mathrm{d} y \exp \left(\mathrm{i} k_{2} \cdot y\right)\left\langle 0\left|\mathrm{~T} S_{\mathrm{rl}}(0) \partial A_{\mathrm{j}}(y)\right| \mathrm{g}_{1} \mathrm{~g}_{2}\right\rangle+\int \mathrm{d} z \exp \left(\mathrm{i} k_{3} \cdot z\right)\left\langle 0\left|\mathrm{~T} S_{\mathrm{rj}}(0) \partial A_{\ell}(z)\right| \mathrm{g}_{1} \mathrm{~g}_{2}\right\rangle .
\end{aligned}
$$

Using gauge invariance it is easy to verify that $k_{1 \mu} R_{\mathrm{rj \ell}}^{\mu}$ is actually of fourth order in the momenta so eq. (7) tells us that, to the order we are working, the $\int \ldots\left\langle 0|\mathrm{~T} \partial A \partial A \partial A| \mathrm{g}_{1} \mathrm{~g}_{2}\right\rangle$ term on the RHS of (7) is just minus the sum of the $\int \ldots\left\langle 0|\mathrm{~T} S \partial A| \mathrm{g}_{1} \mathrm{~g}_{2}\right\rangle$ ones there. To evaluate the last, consider again

$$
\begin{aligned}
& \frac{1}{\mathrm{i}} k_{\mu} \int \mathrm{d} x \exp (\mathrm{i} k \cdot x)\left\langle 0\left|\mathrm{~T} S_{\mathrm{ri}}(0) A_{\mathrm{n}}^{\mu}(x)\right| \mathrm{g}_{1} \mathrm{~g}_{2}\right\rangle \\
& \quad=\int \mathrm{d} x \exp (\mathrm{i} k \cdot x)\left\langle 0\left|\mathrm{~T} S_{\mathrm{ri}}(0) \partial A_{\mathrm{n}}(x)\right| \mathrm{g}_{1} \mathrm{~g}_{2}\right\rangle+\int \mathrm{d} x \exp (\mathrm{i} k \cdot x)\left\langle 0\left|\delta\left(x^{0}\right)\left[A_{\mathrm{n}}^{0}(x), S_{\mathrm{ri}}(0)\right]\right| \mathrm{g}_{1} \mathrm{~g}_{2}\right\rangle .
\end{aligned}
$$

The LHS is of $\mathrm{O}\left((p, k)^{4}\right)$; we may then substitute the RHS into (7) to obtain finally

$\int \mathrm{d} x \mathrm{~d} y \exp \left[\mathrm{i}\left(k_{1} \cdot x+k_{2} \cdot y\right)\right]\left\langle 0\left|\mathrm{~T} \partial A_{\mathrm{r}}(x) \partial A_{\mathrm{j}}(y) \partial A_{\ell}(0)\right| \mathrm{g}_{1} \mathrm{~g}_{2}\right\rangle=\left\langle 0\left|P_{\mathrm{jr} \ell}(0)+P_{\ell \mathrm{rj}}(0)\right| \mathrm{g}_{1} \mathrm{~g}_{2}\right\rangle+\mathrm{O}\left((p, k)^{4}\right)$,

where the $P$ are pseudoscalar operators defined by

$\delta(x) \delta(y) P_{\mathrm{jrl}}(0)=\delta\left(x^{0}-y^{0}\right)\left[A_{\mathrm{j}}^{0}(x), \delta\left(y^{0}\right)\left[A_{\mathrm{r}}^{0}(y), \partial A_{\ell}(0)\right]\right]$.

Evaluating the commutators we can see that if the quark masses are nonvanishing (and only in this case) the $P$ are expressible as

$P_{\mathrm{jr} \ell}=\sum_{\mathrm{q}} c(\mathrm{jr} \ell, \mathrm{q}) 2 \mathrm{i} m_{\mathrm{q}} \overline{\mathrm{q}} \gamma_{\mathrm{s}} \mathrm{q}$,

where the coefficients $c$ depend on quark mass ratios, $m_{\mathrm{q}} / m_{\mathrm{q}}$. Thus the RHS of $(8 \mathrm{a})$ is a combination of terms $\left\langle 0\left|2 \mathrm{i} m_{\mathrm{q}} \overrightarrow{\mathrm{q}}(0) \gamma_{5} \mathrm{q}(0)\right| \mathrm{g}_{1} \mathrm{~g}_{2}\right\rangle$.

These terms contain, in principle poles due to intermediate $\pi^{0}, \eta, \eta^{\prime}, 1$ particles. Of these, the $\eta^{\prime}$, ı poles are suppressed in the chiral limit; the $\pi^{0}, \eta$ ones only enter through mixing with $\eta^{\prime}, \mathbf{l}$ which is of the order of flavor SU (3) breaking. Therefore, these expressions can be obtained, to the order we are interested in, in terms of the standard anomaly, eq. (1). Indeed, $\left\langle 0\left|2 \mathrm{i} m_{\mathrm{q}} \overline{\mathrm{q}} \gamma_{4} \mathrm{q}\right| \mathrm{g}_{1} \mathrm{~g}_{2}\right\rangle=\left(-T_{\mathrm{F}} \alpha_{\mathrm{S}} / 2 \pi\right)\left\langle 0|G \tilde{G}| \mathrm{g}_{1} \mathrm{~g}_{2}\right\rangle$. (It is to be noted that in spite of superficial appearances to the contrary, the result is symmetric under permutations of $r, j, \ell$.) We have succeeded in extracting the RHS of (6), hence $\Phi(0)$, and, in view of (5), the physical scattering amplitude to $\mathrm{O}\left((p, k)^{4}\right)$ corrections. The results (which we have checked by an explicit calculation of the pentagon graph) can best be expressed in the form of effective lagrangians for two gluons with color indices $a, b$, and mesons $r, j$, $\ell$.

$\mathscr{L}_{\text {int }}^{\text {eff }}(\mathrm{r}, \mathrm{j}, \ell)=\left(T_{\mathrm{F}} \alpha_{\mathrm{s}} / 8 \pi f_{\pi}^{3}\right) \lambda(\mathrm{rj} \ell) \phi_{\mathrm{r}} \phi_{\mathrm{j}} \phi_{\ell} \tilde{G} G \delta_{a b}$,

where $\lambda\left(\mathrm{K}^{+} \overline{\mathbf{K}}^{0} \pi^{-}\right)=\lambda\left(\mathrm{K}^{-} \mathrm{K}^{0} \pi^{+}\right)$was given in eq. (3b) and 
$\lambda\left(\mathrm{K}^{+} \mathrm{K}^{-} \pi^{0}\right)=\frac{\left(m_{\mathrm{s}}+m_{\mathrm{u}}\right)^{2}}{2 m_{\mathrm{u}} m_{\mathrm{s}}} \frac{f_{\pi}^{2}}{f_{\mathrm{K}}^{2}}, \lambda\left(\mathrm{K}^{0} \overline{\mathrm{K}}^{0} \pi^{0}\right)=\frac{-\left(m_{\mathrm{s}}+m_{\mathrm{d}}\right)^{2}}{2 m_{\mathrm{s}} m_{\mathrm{d}}} \frac{f_{\pi}^{2}}{f_{\mathrm{K}}^{2}}$

$\lambda\left(\eta \pi^{+} \pi^{-}\right)=\frac{\left(m_{\mathrm{d}}+m_{\mathrm{u}}\right)^{2}}{\sqrt{3} m_{\mathrm{d}} m_{\mathrm{u}}} \frac{f_{\pi}}{f_{\mathrm{\eta}}}, \lambda\left(\eta \pi^{0} \pi^{0}\right)=-\frac{1}{2 !} \frac{4}{\sqrt{3}} \frac{f_{\pi}}{f_{\eta}}$,

$\lambda\left(\pi^{+} \pi^{-} \pi^{0}\right)=\lambda\left(\pi^{0} \pi^{0} \pi^{0}\right)=0$, etc. These lagrangians give the exact mass-shell scattering amplitudes, to corrections $\mathrm{O}\left((p, k)^{4}\right)$. These corrections are of two types: the terms $F_{4}$ in eq. (4), and corrections to $F_{2}$. The first may be seen to be $\mathrm{O}(|\boldsymbol{k}|, M /$ (typical hadronic mass $))$; in all our applications this will be small. In fact, it will vanish in the chiral limit. The corrections to $F_{2}$ may be estimated by writing a dispersion relation for $\Phi_{\text {phys }}$ in the variable $s=\left(p_{\mathrm{a}}+p_{\mathrm{b}}\right)^{2}$, subtracted at $s=0$ where (9) give the exact result. One finds that the only intermediate states that contribute appreciably are the $\eta^{\prime}, 1(1440)$ poles. The expected corrections are of some $50 \%$ for $s=M_{1}^{2}$ and smaller for $s=M_{\eta}^{2}$, so our calculation should give at least the correct order of magnitude.

Before plunging into the applications a few words may be said regarding two important questions. First, because of $\pi^{0}, \eta$ mixing with $\eta^{\prime}, \Phi$ (for example) will have poles at $\left(p_{\mathrm{a}}+p_{\mathrm{b}}\right)^{2}=M_{\pi^{0}}^{2}, M_{\eta}^{2}$. As stated before, these poles give corrections of higher order in SU(3) breaking; in $\eta \pi \pi$ they are small ( $20 \%$ level) in the $\mathrm{KK} \pi$ cases much smaller. Details about these corrections will be found in ref. [3]; here we neglect them. Second, a formula like, say, eq. (3) is surprising: a divergent result would seem to follow if one of the quark masses vanishes. It is not true. Our derivation assumes implicitly and explicitly, and that several times, that quarks are not massless particles. To see what happens, assume that one of the quark masses is small, $\mu$, and that the two others are kept finite, $m$ (and equal to simplify the calculation). A Feynman diagram evaluation gives an amplitude for rj $\ell \rightarrow g g$ of the form $\widetilde{G} G \mu m \int_{0}^{1} \mathrm{~d} x x \int_{0}^{1} \mathrm{~d} y\left[\mu^{2}-2 x(1-x) y p_{\mathrm{a}} \cdot p_{\mathrm{b}}\right]^{-2}$. Thus, if $p_{\mathrm{a}} \cdot p_{\mathrm{b}}$ were exactly zero, we would obtain $\widetilde{G} G m /$ $\mu$ as in eq. (3); if, however, $\mu=0$ to begin with, we get a zero result: the singularity at $\mu=0$ is only apparent. (The same situation arises indeed in the ordinary triangle anomaly.)

A full discussion of these two points will be given in ref. [3].

\section{Phenomenological implications}

We will consider the three meson decays of the 1 and $\eta^{\prime}$ particles. For the $1(1440)$, presumably a pseudoscalar glueball [7] we perform the calculation in the bag model, i.e., we consider the iota to be two free gluons inside a bag of radius $R$. Then,

$\Gamma_{\mathrm{l}}(\mathrm{rj} \ell)=\frac{1-\delta(\mathrm{rj} \ell)}{8 \sqrt{3}} \frac{M_{\mathrm{l}}^{2}\left(M_{\mathrm{l}}-M_{\mathrm{r}}-M_{\mathrm{j}}-M_{\ell}\right)^{2}}{\left(2 R f_{\pi}\right)^{3}\left(4 \pi f_{\pi}\right)^{3}}\left(\frac{T_{\mathrm{F}} \alpha_{\mathrm{s}}}{\pi}\right)^{2} \lambda^{2}(\mathrm{rj} \ell)$,

where $\delta$ are the relativistic corrections to phase space (10-30\%). For numerical evaluations we take $m_{\mathrm{s}} / m_{\mathrm{d}}=20$, $m_{\mathrm{d}} / m_{\mathrm{u}}=2, f_{\mathrm{K}}=1.18 f_{\pi}, f_{\eta}=0.73 f_{\pi}, A=140 \mathrm{MeV}$ for the QCD parameter; and let $\left(2 R f_{\pi}\right)^{3}=\rho$. Then,

$\Gamma_{1}\left(\overline{\mathrm{K}}^{0} \mathrm{~K}^{+} \pi^{-}\right)=\Gamma_{\mathrm{l}}\left(\mathrm{K}^{0} \mathrm{~K}^{-} \pi^{+}\right)=4.6 \mathrm{MeV} / \rho, \quad \Gamma_{1}\left(\mathrm{~K}^{+} \mathrm{K}^{-} \pi^{0}\right)=4.0 \mathrm{MeV} / \rho, \quad \Gamma_{1}\left(\mathrm{~K}^{0} \overline{\mathrm{K}}^{0} \pi^{0}\right)=1.1 \mathrm{MeV} / \rho$,

$\Gamma_{1}\left(\eta \pi^{+} \pi^{-}\right)=0.74 \mathrm{MeV} / \rho, \quad \Gamma_{1}\left(\eta \pi^{0} \pi^{0}\right)=0.3 \mathrm{MeV} / \rho, \quad \Gamma_{1}\left(\pi^{+} \pi^{-} \pi^{0}\right)=\Gamma_{1}\left(\eta \eta \pi^{0}\right)=\Gamma_{1}\left(\pi^{0} \pi^{0} \pi^{0}\right)=0$.

This explains correctly the dominance of the decays into kaons, compared to those involving $\eta$. For the total width we have to decide on a value for $R$, and this is what causes the largest uncertainties in our calculation. Allowing $R^{-1}$ to vary [8] between $145 \mathrm{MeV}$ and $313 \mathrm{MeV}$, we have

$\Gamma_{1}($ all $)=7.3-76 \mathrm{MeV} \quad$ (experimentally, $\Gamma_{1}=76 \mathrm{MeV}$ ).

For the $\eta^{\prime}$ decay we find a formula like (10) substituting $M_{\mathfrak{\imath}}$ by $M_{\eta^{\prime}}$ and multiplying by $\sin ^{2} \theta_{\eta^{\prime} \mathrm{g}}$ for the gluonic component of the $\eta^{\prime}$. With $\sin ^{2} \theta_{\eta^{\prime} g} \sim \frac{1}{2}$, 
$\Gamma_{\eta^{\prime}}^{\text {gluc }}(\eta \pi \pi)=0.02-0.16 \mathrm{MeV} \quad\left(\right.$ experimentally $\left.\Gamma_{\eta}(\eta \pi \pi)=0.18 \mathrm{MeV}\right)$.

(The $\eta^{\prime}$ has a contribution from its quark component, easily evaluated with the techniques of ref. [9] to be $\Gamma_{\eta}^{\text {quark }}(\eta \pi \pi) \simeq 0.04 \mathrm{MeV}$, so $\Gamma_{\eta}^{\text {glue }}$ should really be compared with $\left.\Gamma^{\mathrm{exp}}-\Gamma^{\text {quark }} \simeq 0.14 \mathrm{MeV}\right)$.

We finish this note with a few comments on our phenomenological results. It is clear that we could fit both total widths with reasonable values for $R$ but this we deem a profitless occupation at the present state of the art. We merely prefer to note qualitative agreement in a very wide range of widths, due largely to the values of the $\lambda(\mathrm{rj} \ell)$ :

$\sum_{\mathrm{K}} \lambda^{2}(\mathrm{KK} \pi): \sum_{\pi} \lambda^{2}(\eta \pi \pi) \sim 790: 16$

Particular attention should be drawn to the natural explanation of the large ratio $\Gamma_{1}(\mathrm{KK} \pi) / \Gamma_{1}(\eta \pi \pi)$, contrary to phase space expectations and, as far as we know, not given by any mechanism other than ours.

\section{Acknowledgement}

We are grateful to M.J.G. Veltman whose questions concerning $\eta$ decay are at the root of this work. This research was supported by the US Department of Energy and CAICYT, Spain.

\section{Note added}

The results of this paper cannot be obtained with the method of phenomenological lagrangians. This will be discussed in ref. [3].

\section{References}

[1] J.S. Bell and R. Jackiw, Nuovo Cimento 60A (1969) 47;

S.L. Adler, Phys. Rev. 177 (1969) 2426.

[2] J. Wess and B. Zumino, Phys. Lett. B 37 (1971) 95, and references therein.

[3] R. Akhoury and F.J. Ynduráin, Five-point functions and anomalies, in preparation.

[4] S.L. Adler and W.A. Bardeen, Phys. Rev. 182 (1969) 1517;

W.A. Bardeen, Nucl. Phys. B 75 (1974) 246;

R.J. Crewther, Phys. Rev. Lett. 28 (1972) 1421.

[5] S.U. Chung et al., in: QCD and beyond (Rencontres de Moriond, 1986).

[6] S.L. Adler, Phys. Rev. 137 (1965) B1022;139(1965) B1638.

[7] Particle Data Group, M. Aguilar-Benitez et al., Review of particle properties, Phys. Lett. B 170 (1986) Ic.

[8] P. Hasenfratz et al., in: Topical questions in QCD, Phys. Scr. 23 (1980) 914;

J. Gasser and H. Leutwyler, Phys. Rep. 87 (1982) 77;

N. Isgur, Phys. Rev. D 21 (1980) 779.

[9] F.J. Ynduráin, University of Michigan preprint UM-TH-87-10. 